\title{
Ancestral polymorphism and recent invasion of transposable elements in Drosophila species
}

\author{
Elaine Silva Dias and Claudia Marcia Aparecida Carareto*
}

\begin{abstract}
Background: During the evolution of transposable elements, some processes, such as ancestral polymorphisms and horizontal transfer of sequences between species, can produce incongruences in phylogenies. We investigated the evolutionary history of the transposable elements Bari and 412 in the sequenced genomes of the Drosophila melanogaster group and in the sibling species D. melanogaster and D. simulans using traditional phylogenetic and network approaches.

Results: Maximum likelihood (ML) phylogenetic analyses revealed incongruences and unresolved relationships for both the Bari and 412 elements. The DNA transposon Bari within the D. ananassae genome is more closely related to the element of the melanogaster complex than to the sequence in D. erecta, which is inconsistent with the species phylogeny. Divergence analysis and the comparison of the rate of synonymous substitutions per synonymous site of the Bari and host gene sequences explain the incongruence as an ancestral polymorphism that was inherited stochastically by the derived species. Unresolved relationships were observed in the ML phylogeny of both elements involving D. melanogaster, D. simulans and D. sechellia. A network approach was used to attempt to resolve these relationships. The resulting tree suggests recent transfers of both elements between $D$. melanogaster and D. simulans. The divergence values of the elements between these species support this conclusion.

Conclusions: We showed that ancestral polymorphism and recent invasion of genomes due to introgression or horizontal transfer between species occurred during the evolutionary history of the Bari and 412 elements in the melanogaster group. These invasions likely occurred in Africa during the Pleistocene, before the worldwide expansion of D. melanogaster and D. simulans.
\end{abstract}

Keywords: Transposable elements, Ancestral polymorphism, Horizontal transfer, Introgressive hybridization, Recent invasion, Drosophila melanogaster group

\section{Background}

Transposable elements are segments of repetitive DNA that can mobilize and propagate within host genomes. They have long been considered to be selfish DNA sequences because of the deleterious effects of their mobilization on the host genome. Recent advances in genome analysis methods have revealed the significant contribution of transposable elements to genome evolution as sources of genomic novelty, as they can promote rearrangements [1] and duplications [2] and can produce new regulatory sequences [3] that drive the changes necessary for genome evolution [4]. The emergence of transposable

\footnotetext{
* Correspondence: carareto@ibilce.unesp.br

Department of Biology, São José do Rio Preto, UNESP - São Paulo State University, São Paulo, Brazil
}

(C) 2012 Dias and Carareto; licensee BioMed Central Ltd. This is an Open Access article distributed under the terms of the

elements in a genome can occur in three ways: de novo emergence, by the recombination of existing elements within genomes; horizontal transfer, by a vector; and introgression, by hybridization between two species (one with and one without a given element) [5,6]. The origin of a transposable element in a new genome by the last two processes may produce incongruences when the phylogeny of the elements is compared to those of the species that harbor them. In addition, incongruence can also be produced when two or more variants in an ancestral lineage are stochastically inherited by the derived lineages. Horizontal transfer has been reported in several organisms (for a review see $[7,8]$ ), primarily between closely related species, given the requirement of shared time and space. In many cases, such species also share putative vectors (for a review see [9]); however, the occurrence of 
phylogenetic incongruence due to the stochastic inheritance of ancestral polymorphisms, although potentially common and frequently given as an alternative hypothesis to horizontal transfers [8], is less often demonstrated in the literature.

The genus Drosophila has been the focus of numerous studies involving transposable elements, and the aforementioned processes have been described in these species via bioinformatics analyses and analysis of natural populations $[8,9]$; such studies have focused on species of the melanogaster group, especially the melanogaster subgroup. This subgroup comprises nine species ( $D$. yakuba, D. teissieri, $D$. santomea, $D$. erecta, $D$. orena, $D$. melanogaster, $D$. simulans, $D$. sechellia and D. mauritiana) that differ in many aspects, such as geographical distribution and food and host preference, but that diverged relatively recently. The subgroup is one of ten subgroups of melanogaster, eight of which are found in Asia and three in Africa (melanogaster, montium and ananassae); however, only the melanogaster subgroup is endemic to the Afrotropical region [10]. This subgroup is thought to have originated from a proto-melanogaster founder population that arrived in Africa 17-20 Mya from the Oriental region. This founder population gave rise to the evolutionary lineages that produced the erecta supercomplex approximately 13-15 Mya, the yakuba complex approximately 8-15 Mya and, more recently, the basal lineage of the melanogaster supercomplex. Within this supercomplex, the most basal species, D. melanogaster, arose between 2 and 3 Mya; $D$. simulans, $D$. sechellia and $D$. mauritiana emerged very recently, no more than 0.5 Mya $[11,12]$. D. melanogaster and $D$. simulans are widespread due to very recent global colonization. Also widespread is $D$. ananassae; this species belongs to the ananassae subgroup, the basal clade in the melanogaster species group [13,14] (Species Phylogeny, Additional file 1 Figure S1). This species originated in southeast Asia and subsequently dispersed to other parts of the world, possibly through human activity [15]. The availability of the complete genomes of five species in the subgroup [16] enables the description of numerous transposable element transfers [9]. Meanwhile, the sequencing of just one strain's genome (in four of the five species) and the variable rates of genome coverage can prevent an accurate understanding of the evolutionary history of the elements in these species. One potentially important species is $D$. ananassae, whose genome is available but rarely included in studies. Its widespread distribution, from tropical to subtropical regions, and highly substructured populations make $D$. ananassae a model for studies of genetic variation [17], such as the characterization of transposable elements.

Among the transposable elements studied in the melanogaster subgroup regarding horizontal transmission or introgression are the DNA transposon Bari (transfer between $D$. melanogaster and $D$. simulans [18]), and the retrotransposon 412 (transfers between $D$. melanogaster, D. simulans and D. sechellia $[19,20])$. Bari, a DNA transposon belonging to the Tc1-Mariner superfamily, is an ancient element in the evolutionary lineage of drosophilids that is widespread in both the Drosophila and Sophophora subgenera of the Drosophila genus, although it seems to have been lost in some species [21,22]. Within the genus, there are interspecific structural variations in the terminal-inverted repeats (TIRs), the size of which would have changed over time [23]. Some variants, such as Bari2 (distributed in both Drosophila and Sophophora species) and Bari3 (described in D. willistoni, D. pseudoobscura and D. mojavensis) harbor long TIRs, called LIRs (Long Inverted Repeats), which are approximately 250 bp long. Others, such as Bari1, which is present in the melanogaster complex only, contain short TIRs, called SIRs (Short Inverted Repeats), which are approximately 26-bp long. These three variants, which share over $50 \%$ amino-acid similarity, characterize three subfamilies derived from a common Bari-element ancestor [23]. The element 412, a LTR (Long Terminal Repeats) retrotransposon that belongs to a Gypsy-like superfamily, also seems to have appeared early in the evolution of the Drosophilidae family and to have been subsequently lost in some lineages [24]. In contrast to $D$. melanogaster, the genome of which contains only one 412-subfamily element, $D$. simulans has two intragenomic variants that differ in the size of the 5'LTR UTR regulatory region. These two subfamilies arose from rearrangements and insertion-deletion events that produced new elements that may be capable of escaping host control [24].

Here, we studied the occurrence of both Bari and 412 in the six sequenced genomes of the melanogaster group (D. ananassae, D. erecta, D. yakuba, D. melanogaster, D. simulans and $D$. sechellia). In addition, we compared the in silico results with those obtained from geographic strains of the sibling species D. melanogaster and $D$. simulans to uncover the evolutionary history of these transposable elements in the subgroup. The results will expand our understanding of the processes that shaped their evolution. We showed that at least two Bari variants were present in the ancestral lineage of the melanogaster group and were stochastically inherited, leading to incongruences between the phylogeny of the species and that of the transposable element. We also showed that the transfer of Bari and 412 elements between $D$. simulans and D. melanogaster occurred before the worldwide dispersal of both species and involved only one sequence per element. Thus, ancestral polymorphism, losses and reintroductions can explain the evolutionary distribution of these elements in these species of the melanogaster species subgroup. 


\section{Results}

Using the deposited sequences and the reference sequences for the transposable elements Bari and 412, we searched in the sequenced genomes of the melanogaster group. Homologous full-length and fragmentary sequences of both elements were found in all species (see Table S1 in Additional File 2 and Table S5 in Additional File 3). The fragments were not included in the analyses because most of them contained large deletions and many nucleotide substitutions, which prevent the estimation of the Ks values and the corresponding time of divergence between the sequences. Therefore, only the full-length sequences, with both TIRs for Bari and both LTRs for 412, were used for the analyses.

\section{DNA transposon Bari}

The number of full-length Bari sequences varied by species: 11 were found in $D$. melanogaster, two were found in both $D$. simulans and $D$. sechellia, seven in $D$. erecta and four in $D$. ananassae (see Table S2 in Additional File 2). The only sequence found in D. yakuba was a $215 \mathrm{bp}$ fragment resembling the Bari of D. erecta. The ML reconstruction of evolutionary relationships among the full-length sequences is shown in Figure $1 \mathrm{~A}$ and Additional file 2 Figure $S 2$. The sequences of $D$. ananassae and D. erecta are clustered in well-supported monophyletic clades. Also well-supported is the clade grouping the sequences of the melanogaster complex $(D$. melanogaster, D. simulans and D. sechellia), albeit in a polytomic branch; however, the Bari sequences of $D$. ananassae cluster more closely to those of the melanogaster complex than to those of D. erecta, which is inconsistent with the species phylogeny (Additional File $1)$. The $K 2 p$ distances further contribute to this incongruence, with the sequences of the melanogaster complex and $D$. ananassae being less divergent from each other (melanogaster complex vs. D. ananassae $=0.171 \pm$ 0.012 ; melanogaster complex vs. D. erecta $=0.372 \pm$ $0.024 ; D$. ananassae vs. $D$. erecta $=0.380 \pm 0.023$; see Table S3 in Additional File 2).

Two processes could be responsible for the phylogenetic incongruence observed in the Bari phylogeny: recent invasion of the melanogaster complex by a Bari sequence from $D$. ananassae (or vice versa) or the existence of an ancestral polymorphism followed by stochastic inheritance. To distinguish between these two possibilities, we estimated $K s$, the rate of synonymous substitutions per synonymous site, which provide a measure of divergence in neutral sites, and the time of divergence of the Bari sequences and of the host genes (i.e., $\mathrm{ADH}$ and GAPDH). If the incongruence in the phylogeny is due to differential fixation of Bari variants in the common ancestor and is evolving vertically, then the Ks values of Bari and of the host genes and their time of divergence should be equivalent; however, if the estimates for the transposable element sequences are significantly lower than those for the host genes, then Bari was likely transferred after the species divergence, through horizontal transfer or species hybridization. The sequences of $D$. sechellia and $D$. erecta were not utilized in this analysis because they contained large numbers of stop codons and small deletions. The Bari sequences of $D$. ananassae showed few premature stop codons, all of which were excluded from the alignment, so these sequences were used in the $K s$ estimate. The average $K s$ values of the Bari sequences and host genes were as follows: D. melanogaster vs. D. ananassae, Bari Ks $=0.409 \pm$ 0.049 and host genes $K s=0.428 \pm 0.058 ; D$. simulans vs. D. ananassae, Bari $K s=0.409 \pm 0.047$ and host genes $K s=0.422 \pm 0.055$ (see Tables S4 and S5 in Additional File 2). Using the estimated $K s$ and 0.011 substitutions per site per million years (My) [25] as the rate of synonymous substitution, the average time of divergence of Bari in these species was estimated at $17.68 \mathrm{My}$, and that of the host genes was 19.34 My. During this latter period, the lineages that gave rise to $D$. ananassae and the melanogaster subgroup were still evolving in Asia [10], suggesting that the Bari sequences diverged from a common sequence in the common ancestor. These estimates suggest that the incongruence resulted from stochastic retention of the same Bari1-like variant by the ancestors of $D$. ananassae and of the melanogaster complex. The loss of parts of the TIRs followed, yielding long TIRs in $D$. ananassae and short TIRs in the melanogaster complex [23].

The ML tree did not allow us to resolve the relationships among the Bari1 sequences within the melanogaster complex (D. melanogaster, D. simulans, D. sechellia) because the sequences are very similar and cluster within an unresolved branch. We therefore reconstructed the sequence relationships using a network tree. This approach can resolve relationships among sequences with low diversity and can thus be used to infer the origin of multiple copies from a unique sequence. In addition, the network reveals relationships between ancestral and derived sequences and introduces median vectors, which represent ancestral, lost or unsampled sequences [26,27]; these relationships cannot be inferred from the classical phylogenies.

The network shows a second phylogenetic incongruence in the Bari1 sequences, revealing a closer relationship between the copies of D. simulans and D. melanogaster than either has to $D$. sechellia (Figure 1B). The two full-length sequences of $D$. simulans are directly related to a unique sequence of $D$. melanogaster, which is centrally positioned on the network and is the sequence from which all the other sequences of this species diverged. Moreover, all sequences of $D$. melanogaster are very similar 
A

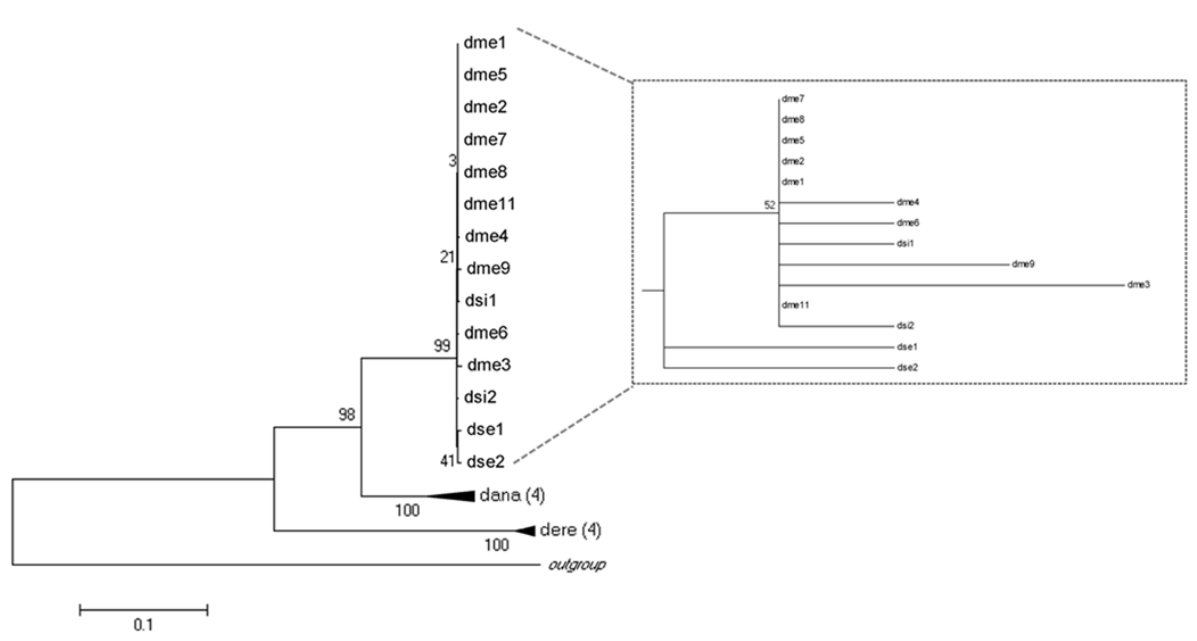

B $\square$ D. melanogaster

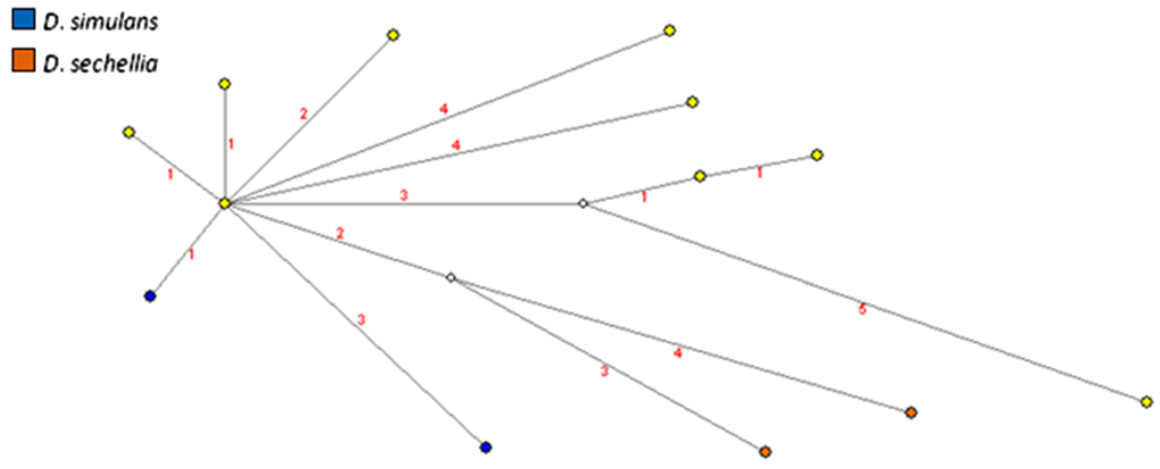

C

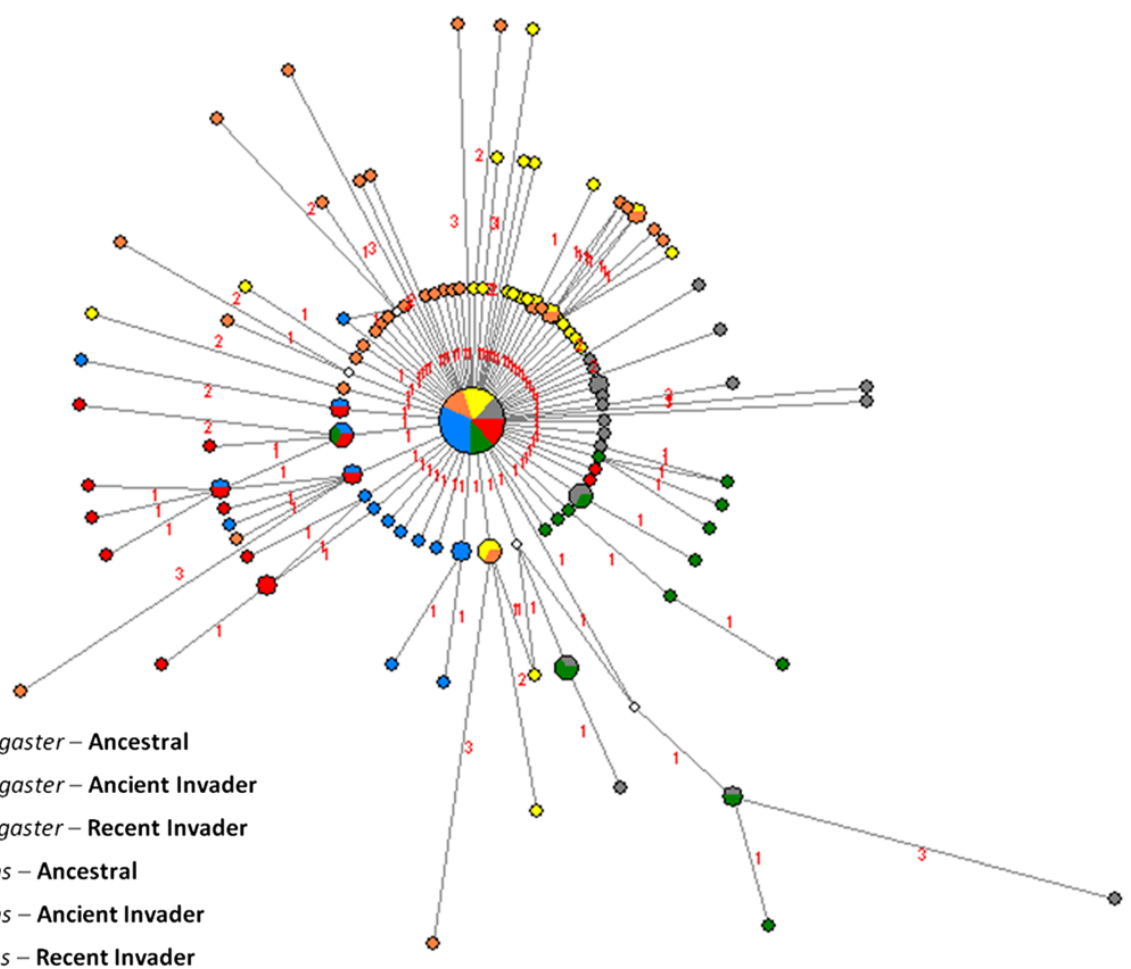

Figure 1 (See legend on next page.) 
(See figure on previous page.)

Figure 1 Phylogenetic reconstructions conducted using sequences of the DNA transposon Bari in the melanogaster group of Drosophila. (A) Phylogenetic analysis by maximum likelihood and (B) Network using the sequences of the transposase of the full-length copies obtained from sequenced genomes of the melanogaster group; (C) Network reconstructed using a region of the transposase sequenced in natural populations of the D. melanogaster and D. simulans species. In the network, full circles correspond to the sampled sequences; empty circles correspond to median vectors ancestral nodes, which represent lost sequences or sequences not sampled. Circle size corresponds to sequence frequency; branch size is proportional to the number of mutations that occurred, as indicated by the numbers above the branches.

$(K 2 p=0.0020 \pm 0.0006)$, suggesting a recent origin. The age of these copies was estimated at $\sim 40,000$ y $(K s=0.00086 \pm$ 0.00084 ), and the longest time of divergence between the Bari1 sequences of $D$. melanogaster and that of $D$. simulans was estimated at $\sim 196,900 \mathrm{y}(K s=0.0004 \pm 0.0004$; average time $=32,800 \mathrm{y}$; shortest time $=0$; see Table S4 in Additional File 2). Because the D. melanogaster and D. simulans lineages split from a common ancestor between 2 and 3 Mya [11,12], the network topology and the divergence time of the Bari1 sequences in both species are inconsistent with the species phylogeny and the estimated divergence time. These data suggest a very recent transfer of Bari1 from $D$. simulans to $D$. melanogaster and further suggest that following this transfer, the sequence remained active and dispersed within the $D$. melanogaster genome, producing the similar copies that are observed today. The short branches and the presence of several similar copies in the D. melanogaster network, all derived from the same sequence, are evidence of transposition burst, a common process after the introduction of a new element into a naïve genome[20], supporting the hypothesis of the transfer from $D$. simulans to $D$. melanogaster.

The similarity of the two D. simulans Bari1 sequences to that of $D$. melanogaster is high, with the sequences differing at only a few sites. To identify whether the proposed recent transfer is exclusive to the sequenced genomes, we sequenced a region of the transposase gene in different strains of both species and reconstructed their relationships (Figure 1C). The strains analyzed represented natural populations of different geographic origins: Africa, the ancestral site; Asia and Europe, continents that were first colonized by both species (ancient invaders); and Brazil, where colonization occurred relatively recently (recent invaders). The evolutionary reconstruction shows a sequence shared among all strains. This central sequence, which likely corresponds to the central sequence depicted in Figure 2B, could be the sequence transferred between the species. The sharing of this sequence among strains of different geographic origins (Africa, Asia, Europe and Brazil) suggests that transfer of Bari occurred before the global dispersal of D. melanogaster.

\section{Retrotransposon 412}

As with Bari, we found different numbers of full-length copies (with both LTRs) of the retrotransposon 412 in different species of the melanogaster group. The smallest numbers were found in D. simulans (2) and D. yakuba (2), followed by D. erecta (8), D. sechellia (11), D. ananassae (14) and D. melanogaster (27; see Table S5 in Additional File 3). The ML phylogeny based on the gag region shows monophyletic branches clustering with the sequences of D. ananassae, D. erecta and D. yakuba with high statistical support (Figure 2A and Additional file 3: Figure S3). Like the species, the D. ananassae 412 was the first to diverge. Next, the ancestral element of D. yakuba and D. erecta diverged, also mirroring the species divergence (Additional File 1); however, in the melanogaster complex, the 412 sequences did not form monophyletic groups within each species. Given the very recent divergence of $D$. melanogaster, $D$. simulans and D. sechellia ( 2 and 3 Mya and 0.5 Mya, respectively $[11,12,28]$, the sequences of 412 could not have had time to coalesce, potentially explaining the unresolved relationships among them. Alternatively, the 412 sequence could have been exchanged between these species.

To resolve the phylogenetic relationships of 412 within the melanogaster complex, we again used the network approach (Figure $2 \mathrm{~B}$ ). The reconstruction shows long and short branches connecting the sequences of D. sechellia, suggesting the presence of old and young copies in this species. These sequences are connected to two copies of $D$. simulans through median vectors (ancestral or unsampled sequences). In $D$. simulans, only two full-length sequences were sampled; these sequences are located in different regions of the network. One is related by a long branch to the old sequences of $D$. sechellia through median vectors, whereas the other is more closely related to all the sequences of $D$. melanogaster. As shown in Figure 2B, all copies of $D$. melanogaster are directly derived from this sequence of $D$. simulans, and all have short branches, indicating a very recent origin. This relationship suggests a transfer of 412 from $D$. simulans to D. melanogaster.

To confirm this pattern in strains derived from several natural populations, we sequenced the integrase region of the 412 element in the same strains of D. melanogaster and D. simulans analyzed for Bari (from Africa, Asia, Europe and South America) and reconstructed the network (Figure 2C). A similar relationship was observed in the strains of both species, where most of the D. melanogaster sequences show short branches and 
A

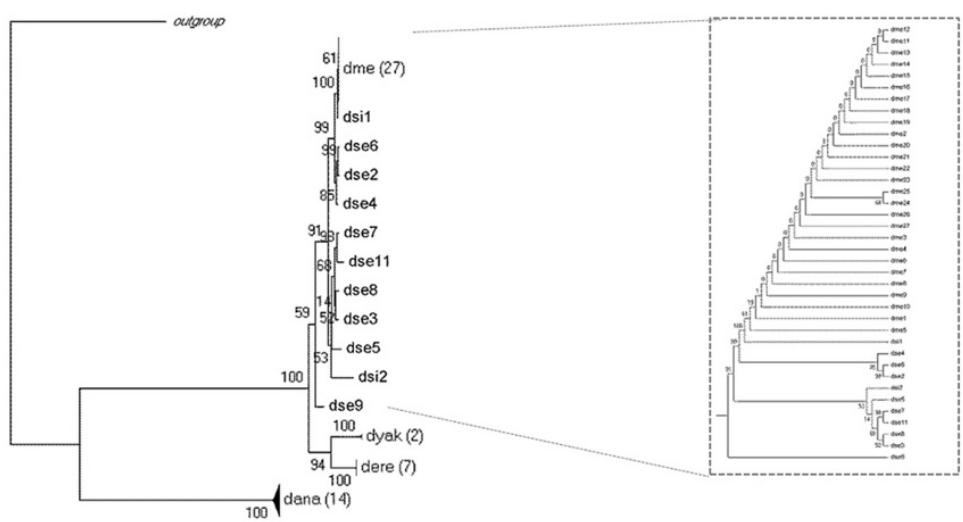

$\stackrel{0.5}{0.5}$

B

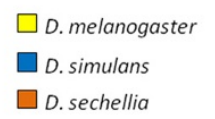

$\square$ D.sechellia

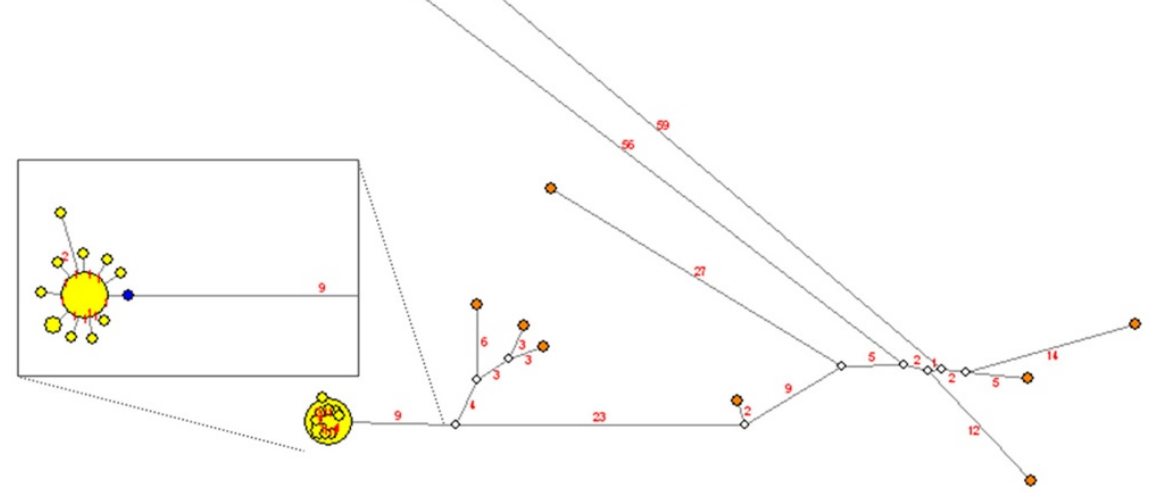

C

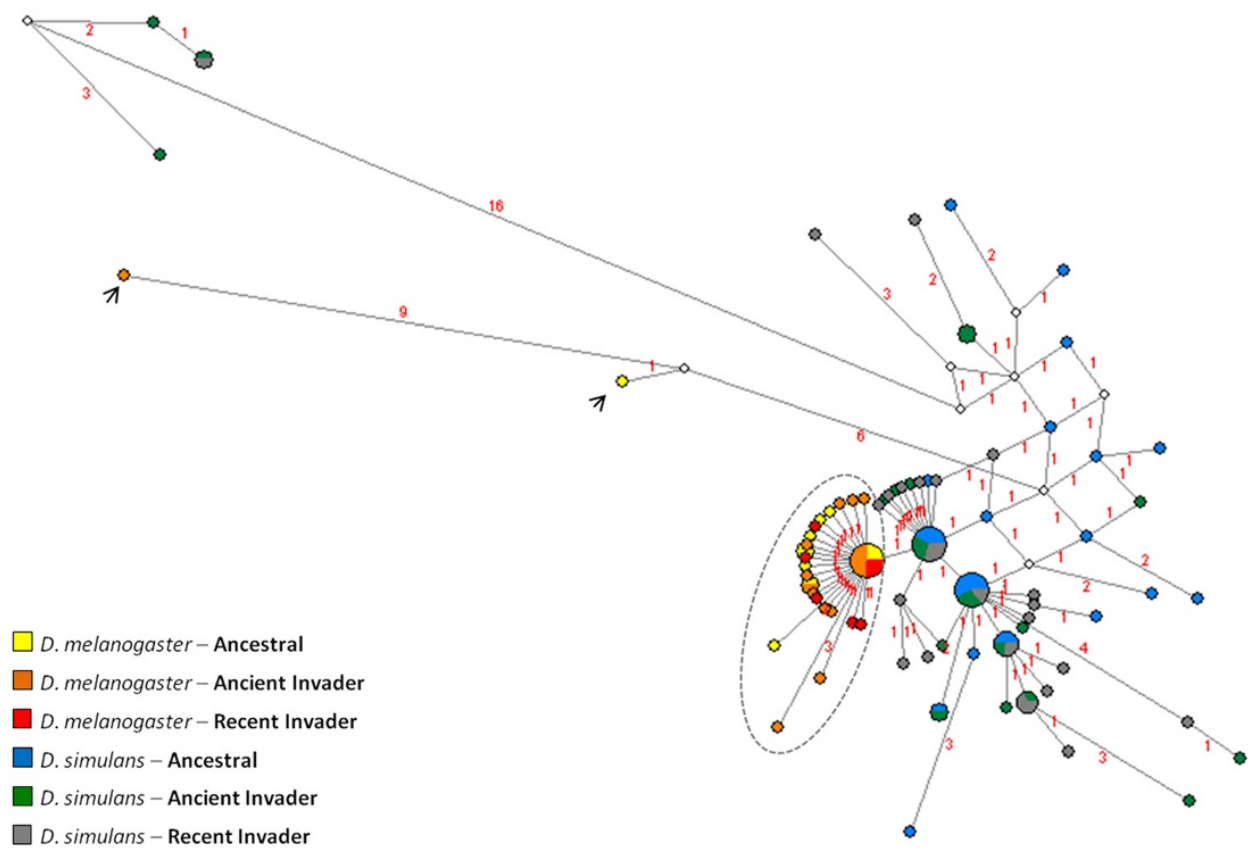

Figure 2 (See legend on next page.) 
(See figure on previous page.)

Figure 2 Phylogenetic reconstructions conducted using the retrotransposon 412 sequences in the melanogaster group of Drosophila. (A) Phylogenetic analysis by maximum likelihood and (B) Network using the gag sequences of full copies obtained from sequenced genomes of the melanogaster group; (C) Network reconstructed using a region of the integrase, sequenced from samples of natural populations of $D$. melanogaster and D. simulans. In the network, full circles correspond to the sampled sequences; empty circles correspond to the ancestral nodes, which represent lost sequences or sequences not sampled. Circle size corresponds to sequence frequency; branch size is proportional to the number of mutations that occurred, as indicated by the numbers above branches.

are derived from only one $D$. simulans sequence. Two sequences of $D$. melanogaster are excluded from this group. They are unresolved and connected by a median vector to the $D$. simulans sequences, as is apparent from the reticulation in the network. There are two main groups of $D$. simulans sequences: one with resolved relationships and short branches, and the other presenting reticulations and several long branches. This scenario reflects the ancient and complex evolutionary history of 412 in this species. Note that the sequence transferred to $D$. melanogaster is shared by all $D$. simulans strains, indicating that it is an active ancestral sequence in this species. In addition, all D. melanogaster strains, regardless of origin, share the sequence derived from $D$. simulans, which differs by only one point mutation. The sharing of ancestral sequences among strains from different continents suggests that, as with Bari, transfer occurred before the dispersal out of Africa.

The age of the proposed transfer was estimated using the molecular clock equation $(\mathrm{t}=K s / 2 \mathrm{r})$. To calculate $K s$, the 27 full-length sequences of $g a g+$ pol ORFs extracted from the sequenced $D$. melanogaster genome were compared to the ancestral sequence from $D$. simulans (Figure 2B, detail). The oldest age estimated is $146,000 \mathrm{y}$ (average $=33,674 \quad \mathrm{y} \pm 0.0084$; lowest $=0.0 \mathrm{y}$ ), suggesting that the transfer of 412 from $D$. simulans to D. melanogaster occurred very recently (see Table S7 in Additional File 3). Indeed, the ages of the insertions in the D. melanogaster genome, as calculated by the divergence between the LTRs of each copy are 94,697 y (the highest) and 0.0 y (the lowest; see Figure S4 in Additional File 3). It is known that at least two factors can introduce biases into this estimation. First, the LTRs evolve, in general, faster than the coding domains of the retrotransposon sequence. Second, because the LTRs of the new copy are synthesized from only one maternal LTR at the moment of reverse transcription, the new LTRs are identical at the point of insertion. This process may conceal the accumulation of divergence between copies. Despite these biases, this approach has been widely used $[29,30]$ and provides useful information about the date of insertion of each copy. The estimated age indicates that transposition would have started soon after the introduction of the copy and continued until very recently, congruent with empirical data and simulations [7]. The analysis shows that all full-length $412 \mathrm{D}$. melanogaster sequences were inserted into the genome no more than 0.1 Mya, while in $D$. simulans, the insertion occurred approximately 0.3 Mya.

\section{Discussion}

The DNA transposon Bari and the retrotransposon 412 are found widely in Drosophila, suggesting a long evolutionary history within the genus $[23,31,32]$. Here, we performed phylogenetic analyses involving both traditional and network approaches that allowed us to reveal the occurrence of ancestral polymorphism and recent transfer of transposable elements between $D$. melanogaster and D. simulans.

\section{Ancestral polymorphism}

We performed an in silico search for the DNA transposon Bari in the sequenced genome of species of the melanogaster group, and we sequenced a region of the transposase in different geographic strains of D. melanogaster and D. simulans. The element exhibits structural variations related to its TIRs, which characterize various Bari subfamilies [23]. Both long and short TIRs were observed in the sequences analyzed in our study. The sequences of elements found in $D$. ananassae and $D$. erecta, which contain long TIRs, included stop codons and are therefore inactive, whereas the sequences of $D$. melanogaster, D. simulans and D. sechellia included short TIRs. In D. melanogaster and D. simulans, there were full-length sequences without stop codons, which therefore suggest putatively active copies. In contrast, the full-length sequences in $D$. sechellia included multiple stop codons.

The element found in D. ananassae, with long TIRs, is more closely related to the element of $D$. melanogaster, which has short TIRs, than to that of D. erecta, which also has long TIRs. These relationships produce incongruences between the element and species phylogenies. The element found in D. erecta (Bari2 subfamily) is widely distributed in Drosophila, whereas those in $D$. ananassae (Bari1-like subfamily) and in the melanogaster complex (Bari1 subfamily) are restricted to their respective species; this pattern indicates that the Bari element of $D$. erecta is older than that of $D$. ananassae [23]. Therefore, we propose that the ancestor of the melanogaster species group possessed at least two Bari variants that were stochastically inherited by the derived 
species. The estimated age of the common ancestor of the Bari sequences in the genomes of $D$. ananassae and D. melanogaster ( $17 \mathrm{Mya})$ is similar to the ages of their host genes ( 19 Mya). During this period, the proto-melanogaster lineage was still diversifying from its sister subgroups in Africa. The only published estimate for the diversification between the melanogaster and ananassae subgroups is $\sim 44$ Mya [25], but it could have occurred more recently, as all of the divergence times estimated in that study are more than two times higher than other estimates (e.g., between the melanogaster and montium subgroups, 41.3 Mya [25] and 12.7 Mya [33] and between D. melanogaster and D. simulans, 5.4 Mya [25], 2.3 Mya [33] and 2-3 Mya [11]). In conclusion, i) the phylogenetic incongruence arising from clustering the Bari sequences of $D$. ananassae with those of the melanogaster complex and ii) the older estimated age of their Bari ancestor with respect to the age of diversification of the melanogaster subgroup and the migration of its ancestral lineage to Tropical Africa support the hypothesis of vertical inheritance with stochastic retention of polymorphic sequences of Bari in these species. The ancestral polymorphism hypothesis is also supported by the smaller distances between the elements of the $D$. ananassae vs. melanogaster complex than between those of the D. erecta vs. melanogaster complex.

As described in the Background section, few reports clearly demonstrate retention of ancestral polymorphisms. One such study examines the DNA transposon mariner, which occurs in the melanogaster subgroup in D. simulans, D. sechellia, D. mauritiana, D. yakuba and D. teissieri, but not in D. melanogaster, D. erecta and D. orena. It is proposed that mariner was present in the ancestral species prior to the radiation of the melanogaster species subgroup and that the element was lost independently in the lineages leading to $D$. melanogaster and $D$. orena - D. erecta. In addition, the mariner sequences of $D$. simulans and D. mauritiana share active copies, a subset of all mariner sequences, that cluster together rather than according to the species phylogeny. This shared polymorphism in populations of $D$. simulans worldwide and in D. mauritiana indicates retention of ancestral polymorphisms [34]. In both the mariner study and in ours, the rates of evolution of the DNA transposon and of a host gene were compared to test the ancestral-polymorphism hypothesis and to explain incongruence between the phylogenies of the species and the transposable element.

\section{Transposable element recent invasion}

The incongruence between the species phylogeny and the phylogenies of the Bari and 412 elements, along with the ages of the sequences shared between $D$. melanogaster and $D$. simulans (less than the age of the species divergence), could arise through either horizontal transfer or introgressive hybridization. An increasing number of reports in the last two decades (mostly published following the rise of large-scale genome sequencing, which allows analysis of most copies from a given genome as well as broader comparative evolutionary analysis) suggest that horizontal transfer of transposable elements occurs frequently in eukaryotes (for a review see [7]), especially in Drosophila (for a review see $[8,9]$ ). Particularly for $D$. melanogaster and $D$. simulans, evidence of horizontal transfer is accumulating in the literature [19,35-42], including the elements Bari $[19,37]$ and 412 $[19,37,42]$, the focus of this study; however, D. melanogaster and $D$. simulans can hybridize, even today, in both the laboratory [43] and in nature [44]; therefore, it is also possible to introduce transposable elements via hybridization.

In order for either horizontal transfer or introgression to occur, species must overlap in time, space and habitat. Moreover, for horizontal transfer, a shared potential vector (e.g., a virus or endobacterium) is required. Currently, the sibling species $D$. melanogaster and $D$. simulans are cosmopolitan and are sympatric in many parts of the world; however, our analyses suggest that transfer events occurred before their worldwide expansion but after species divergence. It is thought that $D$. melanogaster was the first species to diverge from a common ancestor in West Africa and that the ancestor of $D$. simulans, the protosimulans lineage, migrated to east Africa and occupied the Pacific islands and then diversified. After the divergence, $D$. simulans returned to the mainland and expanded, coming into contact with $D$. melanogaster populations in Africa during the Late Pleistocene (around 120 and 9 thousand years ago) [10]; we estimate that transfer of both elements occurred during this period. Overlaps in space, time, and most likely niche, may have provided the necessary conditions for both horizontal transfer and introgression. Regardless of the precise mechanism, after the transfers occurred, both species expanded out of Africa, D. melanogaster with the Homo sapiens migration and $D$. simulans more recently, most likely during the great navigations [12].

The presence of defective fragments of Bari and 412 elements in the sequenced genomes of both species and the presence of two more divergent sequences of the retrotransposon 412 in populations of D. melanogaster (as shown in the network) indicate that both elements were present in the common ancestor of these species and were inherited by both species. The presence of full-length and putatively active copies in D. melanogaster, which were derived exclusively from sequences transferred from $D$. simulans, suggests that $D$. melanogaster either did not inherit active copies of both Bari and 412 from a common ancestor or lost these copies 
early in its diversification. The defective fragments that are still present today would then be remnants of the copies inherited from its ancestor. After the reintroductions of Bari and 412, the transferred sequences remained active in $D$. melanogaster, giving rise to the majority of copies that are currently present in this species. We show here that the amplification of these copies occurred in a short period of time and at elevated rates, resulting in a burst of transposition. This process can be deduced from the network by the presence of identical sequences clustered in nodes or by similar sequences connected by short branches; these characteristics are observed in both species, in both the sequenced genomes and in natural populations. Bursts of transposition have been previously reported, in silico, for other elements in the genomes of the melanogaster subgroup, such as the Helitron DINE-1 in D. yakuba and $D$. ananassae [45] and numerous LTR retrotransposons in the D. melanogaster euchromatin [20].

The element 412 occurs in two subfamilies in $D$. simulans. Only one is observed in D. melanogaster and is very similar to one of the subfamilies found in $D$. simulans [24,30]. Therefore, according to our data, an ancestral sequence of the melanogaster complex was likely inherited by both species, but diversification occurred only in $D$. simulans; later, one of the two subfamilies was transferred to D. melanogaster, which at the time had only defective copies derived from its ancestral lineage. The subfamilies present in D. simulans were the result of rearrangements, indels and point mutations in the regulatory sequences in the $5^{\prime}$ LTR -UTR region; then, these changes gave rise to elements that were able to overcome the host control for transposition and thus able to became invaders $[24,30]$. This process may explain why the retrotransposon 412 remained active in $D$. simulans following its divergence and retained its capacity for amplification following its transfer to D. melanogaster, whose control host system, which had coevolved related with the sequences inherited from the ancestral copies, could not recognize this new element. Data from the literature (reviewed in [9]) suggest that several elements have been independently transferred between the two species over time (e.g., Copia, tirant, Opus, Gypsy 2, Gypsy 5, Gypsy 6, 297, 17.6), but several others may have been transferred simultaneously and very recently (e.g., 412, Blood, Stalker 2, Transpac, Flea), as can be deduced from the very similar ages of the transfers. There is a consensus that multiple elements have recently arrived in D. melanogaster; however, their origins either have not been suggested $[20,23]$ or were not clearly demonstrated $[38,46]$. Utilization of the network approach allowed us to propose, at least for Bari and 412, that D. simulans was the donor species.

\section{Conclusions}

The results obtained here allowed us to propose that the incongruences observed in the phylogeny of the Bari and 412 elements were a result of ancestral polymorphism as well as recent invasion of $D$. melanogaster genome by these elements. The ancestral polymorphism associated with Bari is supported by phylogenetic incongruence, and by a divergence time of Bari between the D. melanogaster complex and $D$. ananassae similar to that of the host genes. The hypothesis of recent invasion of both elements is supported by phylogenetic incongruences revealed by network trees; in addition, the shortest time of divergence is found between the transposable sequences, rather than between the species involved. Moreover, $D$. simulans is thought to have transferred sequences of both elements to $D$. melanogaster. This species in turn would not have inherited or would have lost the active copy that existed in its ancestor as soon as it diverged, and all of its full-length sequences would have been derived from the sequence that was transferred from its sibling species. This introduction would have occurred in Africa before the worldwide expansion of the species, most likely in the late Pleistocene, during which $D$. melanogaster and $D$. simulans returned to sympatry in Africa after diversification in allopatry. In $D$. melanogaster, the elements would be passed through a burst of transposition, producing a high number of fulllength copies over several thousand years.

\section{Methods}

\section{In silico analyses}

The search for copies of the retrotransposon 412 and of the DNA transposon Bari in the sequenced genomes of species of Drosophila melanogaster group (release 5.18 of D. melanogaster and 1.3 for all other species [16]) was performed using BLASTn [47]. The deposited sequences (Repbase databank [48]) described in D. melanogaster were used directly to search in this species. The Bari sequence used [GenBank: X67681] is 1,728 bp long and encodes a 340 aa transposase. The 412 sequence [GenBank: X04132] is 8,039 bp long and encodes a 452 aa gag-like protein and a 1,001 aa pol-like polyprotein (Reverse transcriptase, RNase $\mathrm{H}$ and Intregrase, respectively). For the other species, the deposited sequences were used to identify the reference sequence, i.e., the most complete and conserved sequence of each element in each species. These sequences were used to search for complete and incomplete copies in each genome. The complete copies were tested for the presence and integrity of the transposase gene (Bari) and gag and pol (412) using the software ORF Finder [49]. In the search, the hits with the smallest e-values $\left(>10^{-5}\right)$ and highest RM scores $(>225)$ were selected. Alignment, reconstruction of the phylogeny by maximum likelihood (ML), calculation of the rate of synonymous substitutions per 
Table 1 Strains of $D$. melanogaster and $D$. simulans used in this study

\begin{tabular}{|c|c|c|c|c|c|}
\hline \multirow[t]{2}{*}{ Species } & \multirow{2}{*}{$\begin{array}{l}\text { Classification } \\
\text { with regard to } \\
\text { the origin }\end{array}$} & \multirow[t]{2}{*}{ Geographic Origin } & \multirow{2}{*}{$\begin{array}{l}\text { Collector/ } \\
\text { Stock }\end{array}$} & \multicolumn{2}{|c|}{ GenBank Accessions } \\
\hline & & & & Bari-1 & 412 \\
\hline \multirow[t]{5}{*}{ D. melanogaster } & Ancestral & Madagascar - Africa & David, J & JX140191-JX140203 & JX140342-JX140346 \\
\hline & & Congo - Africa & $14021-0231.24$ & JX140204-JX140220 & JX140347-JX140352 \\
\hline & Ancient Invader & Draveil - France & David, J & JX140221-JX140237 & JX140353-JX140361 \\
\hline & & Delhi - Asia & David, J & JX140238-JX140256 & JX140362-JX140368 \\
\hline & Recent Invader & Florianopolis - Brazil & Granzotto, A & JX140257-JX140275 & JX140369-JX140375 \\
\hline \multirow[t]{6}{*}{ D. simulans } & Ancestral & Madagascar - Africa & David, J & JX140276-JX140288 & JX140376-JX140389 \\
\hline & & Zimbabwe - Africa & Begun, D & JX140289-JX140299 & JX140390-JX140399 \\
\hline & Ancient Invader & Draveil - France & Capy, P & JX140300-JX140307 & JX140400-JX140411 \\
\hline & & Gorak - New Guinea & $14021-0251.009$ & JX140308-JX140318 & JX140412-JX140420 \\
\hline & Recent Invader & Florianopolis - Brazil & Granzotto, A & JX140319-JX140332 & JX140421-JX140435 \\
\hline & & Pernambuco - Brazil & Rohde, C & JX140333-JX140341 & JX140436-JX140445 \\
\hline
\end{tabular}

Classification with the regard to geographic origin of the strains, name of the collectors or stock numbers and GenBank sequence accession numbers.

synonymous site $(K s)$ and analysis of the Kimura 2parameters distance $(K 2 p)$ [50] were performed only for full-length sequences with both TIRs and LTRs, using the package Mega5 [51]. The evolutionary relationships between sequences were also reconstructed using the package Network [52]. The ages of the transposable elements were estimated using the molecular clock equation $\mathrm{r}=\mathrm{k} / 2 \mathrm{~T}$, where $r$ is the rate of neutral synonymous substitution in the genus Drosophila ( $\mathrm{r}=0.011 / \mathrm{site} /$ million years [25] and $k$ is the rate of divergence in the synonymous sites $(K s)$. The molecular-clock hypothesis assumes that when genes from different species are compared, the number of nucleotide changes is proportional to the speciation time. We then estimated the divergence time between species of the melanogaster group using the $K s$ values of the CDS of two host genes (ADH: Alcohol dehydrogenase and GAPDH: Glyceraldehyde 3 phosphate dehydrogenase 1 ; see Table $5 \mathrm{~S}$ in Additional File 2) and of the Bari transposase. The ages of the insertions of the retrotransposon 412 were estimated using the date of divergence between both LTRs of each copy by $K 2 p$ in the molecular clock equation.

\section{Molecular analyses}

The phylogenetic relationships between the Bari sequences of strains of $D$. melanogaster and D. simulans of different geographic origin (Table 1) were also reconstructed. These strains were classified as ancestors (sampled in Africa) or invaders (ancient, sampled in Asia and Europe; or recent, sampled in Brazil) according to place of origin and literature reports [10].

Genomic DNA was extracted from 50 individuals [53]. DNA concentration and integrity was analyzed via spectrophotometer (NanoDrop). Amplification (PCR) was performed using specific primers that anneals to nucleotides 412 to 1,133 (total length of $722 \mathrm{bp}$ ) in the Bari

transposase gene (Bari_F 5' CGG GCT GGT ATT GTT GCT AGG TTT 3' and Bari_R 5' ATC CTA CCC TTA TGG CAT GGA GCA 3') and to nucleotides 5,622 - to 6,499 (total length of $878 \mathrm{bp}$ ) in the 412 integrase gene (412_F 5' TGG SCR AGG TCA WAR GAC AT 3' and 412_R 5' RCT TTS TAT STT ATA GGG CC 3'), 0.625 unit of Taq polymerase (Invitrogen), $200 \mathrm{ng}$ genomic DNA, $1 \mathrm{mM}$ of $\mathrm{MgCl}_{2}, 1 \mathrm{X}$ buffer, $0.08 \mathrm{mM}$ of dNTPs and $0.4 \mathrm{mM}$ of each primer, for a final volume of $25 \mu \mathrm{L}$. PCR conditions were as follows: initial denaturation $\left(94^{\circ} \mathrm{C}\right.$, $120 \mathrm{~s}$ ); followed by 30 cycles of denaturation $\left(94^{\circ} \mathrm{C}, 45 \mathrm{~s}\right)$, annealing $\left(69^{\circ} \mathrm{C}\right.$ for Bari and $59^{\circ} \mathrm{C}$ for $\left.412,45 \mathrm{~s}\right)$ and extension $\left(72^{\circ} \mathrm{C}, 60 \mathrm{~s}\right)$. Each PCR product was analyzed by gel electrophoresis on a $1.0 \%$ agarose gel, purified (DNA GFX DNA \& Gel Band, GE) and cloned (TOPO TA Cloning kit, Invitrogen) according to the manufacturer's specifications. Approximately 30 (D. melanogaster) and 20 (D. simulans) clones were selected for plasmid extraction by phenol/ chloroform protocol and sequenced using the universal primers M13F and M13R. The sequences produced were deposited in the GenBank database (Table 1).

\section{Additional files}

Additional File 1: Figure S1: the phylogenetic relationships
between species of the melanogaster group of Drosophila.

Additional File 2: Tables and figure about the characteristics and evolutionary analyses of the DNA transposon Bari sequences found in the sequenced genomes of species of the melanogaster group of Drosophila. Table corresponding to the $K$ s analyses of the genes $A D H$ and GAPDH.

Additional File 3: Tables and figures about the characteristics and evolutionary analyses of the sequences of the retrotransposon 412 found in the sequenced genomes of species of the melanogaster group of Drosophila.

Competing interests

The authors declare that they have no competing interests. 


\section{Author's contributions}

ESD conducted the bioinformatics and molecular analyses, and CMAC conceived and coordinated the project; both authors wrote the manuscript. Both authors read and approved the final manuscript.

\section{Acknowledgements}

Funding for this project was provided by the Brazilian agencies FAPESP (Fundação de Amparo à Pesquisa do Estado de São Paulo - Grant 2010/ 10731-4 to CMAC and fellowship 2008/07629-3 to ESD), CNPq (Conselho Nacional de Desenvolvimento Científico e Tecnológico; Grant 304880/2009-4 to CMAC) and FUNDUNESP (Fundação para o Desenvolvimento da UNESP; Grant 670/10).

Received: 9 December 2011 Accepted: 10 July 2012

Published: 23 July 2012

\section{References}

1. Lerman D, Michalak P, Helin A, Bettencourt B, Feder M: Modification of heat-shock gene expression in Drosophila melanogaster populations via transposable elements. Mol Biol Evol 2003, 20:135-144.

2. Bejerano G, Lowe C, Ahituv N, King B, Siepel A, Salama S, Rubin E, Kent W Haussler D: A distal enhancer and an ultraconserved exon are derived from a novel retroposon. Nature 2006, 441:87-90.

3. Feschotte C: Opinion - Transposable elements and the evolution of regulatory networks. Nat Rev Genet 2008, 9:397-405.

4. Nakayashiki H: The Trickster in the genome: contribution and control of transposable elements. Genes Cells 2011, 16:827-841.

5. Almeida LM, Carareto CMA: Origem, Proliferação e Extinção de Elementos de Transponíveis: Qual Seria a Importância da Transferência Horizontal na Manutenção desse Ciclo? Série Monografias, SBG, Ribeirão Preto 2005, 1:1-40.

6. Capy P, Langin T, Anxolabehere D, Bazin C: Dynamics and Evolution of Transposable Elements. 1st edition. Austin: Landes Bioscience; 1998.

7. Schaack S, Gilbert C, Feschotte C: Promiscuous DNA: horizontal transfer of transposable elements and why it matters for eukaryotic evolution. Trends Ecol Evol 2010, 25:537-546.

8. Loreto ELS, Carareto CMA, Capy P: Revisiting horizontal transfer of transposable elements in Drosophila. Heredity 2008, 100:545-554.

9. Carareto C: Tropical Africa as a cradle for horizontal transfers of transposable elements between species of the genus Drosophila and Zaprionus. Mobile Genetics Elements 2011, 2:179-186.

10. Lachaise D, Cariou ML, David JR, Lemeunier F, Tsacas L, Ashburner M: Historical biogeography of the Drosophila melanogaster species subgroup. Evolutionary Biology 1988, 22:159-225

11. Hey J, Kliman RM: Population Genetics and phylogenetics of DNA sequence variation at multiple loci within the Drosophila melanogaster species complex. Mol Biol Evol 1993, 10:804-822.

12. Lachaise D, Silvain J: How two Afrotropical endemics made two cosmopolitan human commensals: the Drosophila melanogaster-D. simulans palaeogeographic riddle. Genetica 2004, 120:17-39.

13. van der Linde K, Houle D: A supertree analysis and literature review of the genus Drosophila and closely related genera (Diptera, Drosophilidae). Insect Systematics \& Evolution 2008, 39:241-267.

14. Yang Y, Hou Z-C, Qian Y-H, Kang H, Zeng Q-T: Increasing the data size to accurately reconstruct the phylogenetic relationships between nine subgroups of the Drosophila melanogaster species group (Drosophilidae, Diptera). Mol Phylogenet Evol 2012, 62:214-223.

15. Dobzhansky T, Dreyfus A: Chromosomal aberrations in Brazilian Drosophila ananassae. Proc Natl Acad Sci 1943, 29:301-305.

16. Clark AG, Eisen MB, Smith DR, Bergman CM, Oliver B, Markow TA, Kaufman TC, Kellis M, Gelbart W, lyer VN, et al: Evolution of genes and genomes on the Drosophila phylogeny. Nature 2007, 450:203-218.

17. Das A: Population genomic and bioinformatic studies reveal evolutionary history of Drosophila ananassae. Curr Sci 2005, 89:1316-1321

18. Sanchez-Gracia A, Maside X, Charlesworth B: High rate of horizontal transfer of transposable elements in Drosophila. Trends Genet 2005 24:200-203.

19. Bartolome C, Bello X, Maside X: Widespread evidence for horizontal transfer of transposable elements across Drosophila genomes. Genome Biol 2009, 10:R22.
20. Lerat E, Burlet N, Biémont C, Vieira C: Comparative analysis of transposable elements in the melanogaster subgroup sequenced genomes. Gene 2010, 473:100-109

21. Caizzi R, Caggese C, Pimpinelli S: Bari-1, a new transposon-like family in Drosophila melanogaster with a unique heterochromatic organization. Genetics 1993, 133:335-345.

22. Moschetti R, Caggese C, Barsanti P, Caizzi R: Intra- and interspecies variation among Bari-1 elements of the melanogaster species group. Genetics 1998, 150:239-250

23. Moschetti R, Chlamydas S, Marsano R, Caizzi R: Conserved motifs and dynamic aspects of the terminal inverted repeat organization within Bari-like transposons. Mol Genet Genomics 2008, 279:451-461.

24. Mugnier N, Biemont C, Vieira C: New regulatory regions of Drosophila 412 retrotransposable element generated by recombination. Mol Biol Evol 2005, 22:747-757

25. Tamura K, Subramanian S, Kumar S: Temporal Patterns of Fruit Fly (Drosophila) Evolution Revealed by Mutation Clocks. Mol Biol Evol 2004, 21:36-44.

26. Posada $D, C$ randall $K$ : Intraspecific gene genealogies: trees grafting into networks. Trends Ecol Evol 2001, 16:37-45.

27. Cordaux R, Hedges DJ, Batzer MA: Retrotransposition of Alu elements: how many sources? Trends Genet 2004, 20:464-467.

28. Da Lage J, Kergoat G, Maczkowiak F, Silvain J, Cariou M, Lachaise D: A phylogeny of Drosophilidae using the Amyrel gene: questioning the Drosophila melanogaster species group boundaries. Journal of Zoological Systematics and Evolutionary Research 2007, 45:47-63.

29. Bowen N, McDonald J: Drosophila Euchromatic LTR Retrotransposons are Much Younger Than the Host Species in Which They Reside. Genome Res 2001, 11:1527-1540.

30. Mugnier N, Gueguen L, Vieira C, Biemont C: The heterochromatic copies of the LTR retrotransposons as a record of the genomic events that have shaped the Drosophila melanogaster genome. Gene 2008, 411:87-93.

31. Cizeron G, Lemeunier F, Loevenbruck C, Brehm A, Biemont C: Distribution of the retrotransposable element 412 in Drosophila species. Mol Biol Evol 1998, 15:1589-1599.

32. Caggese C, Pimpinelli S, Barsanti P, Caizzi R: The distribution of the transposable element Bari-1 in the Drosophila melanogaster and Drosophila simulans genomes. Genetica 1995, 96:269-283.

33. Russo C, Takezaki N, Nei M: Molecular phylogeny and divergence times of Drosophilid species. Mol Biol Evol 1995, 12:391-404.

34. Maruyama K, Hartl DL: Evidence for interspecific transfer of the transposable element mariner between Drosophila and Zaprionus. J Mol Evol 1991, 33:514-524.

35. Bregliano JC, Kidwell MG (Eds): Hybrid dysgenesis determinants. New York: Academic Pres; 1983

36. Jordan IK, Matyunina LV, McDonald JF: Evidence for the recent horizontal transfer of long terminal repeat retrotransposon. Proc Natl Acad Sci USA 1999, 96:12621-12625

37. Sanchez-Gracia A, Maside X, Charlesworth B: High rate of horizontal transfer of transposable elements in Drosophila. Trends Genet 2005, 21:200-203.

38. Ludwig A, Valente V, Loreto ELS: Multiple invasions of Errantivirus in the genus Drosophila. Insect Mol Biol 2008, 17:113-124.

39. Vidal NM, Ludwig A, Loreto ELS: Evolution of Tom, 297, 17.6 and rover retrotransposons in Drosophilidae species. Mol Genet Genomics 2009, 282:351-362.

40. Depra M, Valente VLD, Margis R, Loreto ELS: The hobo transposon and hobo-related elements are expressed as developmental genes in Drosophila. Gene 2009, 448:57-63.

41. de Setta N, Van Sluys MA, Capy P, Carareto CMA: Multiple invasions of Gypsy and Micropia retroelements in genus Zaprionus and melanogaster subgroup of the genus Drosophila. BMC Evol Biol 2009, 9:279.

42. Lerat $\mathrm{E}$, Burlet $\mathrm{N}$, Biemont $\mathrm{C}$, Vieira C: Comparative analysis of transposable elements in the melanogaster subgroup sequenced genomes. Gene 2011, 473:100-109.

43. de Lucca M, Carareto C, Ceron C: Distribution of the Bari-I transposable element in stable hybrid strains between Drosophila melanogaster and Drosophila simulans and in Brazilian populations of these species. Genetics and Molecular Biology 2007, 30:676-680.

44. Sperlich D: Hybrids between Drosophila melanogaster and D. simulans in nature. Drosophila Information Service 1962, 36:118. 
45. Yang HP, Hung TL, You TL, Yang TH: Genomewide comparative analysis of the highly abundant transposable element DINE-1 suggests a recent transpositional burst in Drosophila yakuba. Genetics 2006, 173:189-196.

46. Heredia F, Loreto ELS, Valente VLS: Complex evolution of gypsy in drosophilid species. Mol Biol Evol 2004, 21:1831-1842.

47. Altschul SF, Gish W, Miller W, Myers EW, Lipman DJ: Basic local alignment search tool. J Mol Biol 1990, 215:403-410.

48. Jurka J, Kapitonov W, Pavlicek A, Klonowski P, Kohany O, Walichiewicz J: Repbase update, a database of eukaryotic repetitive elements. Cytogenet Genome Res 2005, 110:462-467.

49. Open Reading Frame Finder. http://www.ncbi.nlm.nih.gov/gorf/gorf.html.

50. Kimura M: A simple method for estimating evolutionary rates of base substitutions through comparative studies of nucleotide sequences. Journal of Molecular Evolution 1980, 16:111-120.

51. Tamura K, Peterson D, Peterson N, Stecher G, Nei M, Kumar S: MEGA5: molecular evolutionary genetics analysis using maximum likelihood, evolutionary distance, and maximum parsimony methods. Mol Biol Evol 2011, 28:2731-2739.

52. Bandelt $H$, Forster $P$, Rohl $A$ : Median-joining networks for inferring intraspecific phylogenies. Mol Biol Evol 1999, 16:37-48.

53. Jowett T: Preparation of nucleic acids. In In Drosophila: A Practical Approach. Edited by Roberts DB. Oxford: IRL Press; 1986:275-285.

doi:10.1186/1471-2148-12-119

Cite this article as: Dias and Carareto: Ancestral polymorphism and recent invasion of transposable elements in Drosophila species. BMC Evolutionary Biology 2012 12:119.

\section{Submit your next manuscript to BioMed Central and take full advantage of:}

- Convenient online submission

- Thorough peer review

- No space constraints or color figure charges

- Immediate publication on acceptance

- Inclusion in PubMed, CAS, Scopus and Google Scholar

- Research which is freely available for redistribution 